\title{
Investigation of myo-Inositol Catabolism in Rhizobium leguminosarum bv. viciae and Its Effect on Nodulation Competitiveness
}

\author{
Judith Fry, Martin Wood, and Philip S. Poole \\ Division of Microbiology School of Animal and Microbial Sciences and Department of Soil Science, \\ University of Reading, Reading RG6 6AJ, U.K. \\ Submitted 24 December 2000; Accepted 25 April 2001.
}

Three discrete loci required for growth on myo-inositol in Rhizobium leguminosarum bv. viciae have been characterized. Two of these are catabolic loci that code for malonate semialdehyde dehydrogenase (iolA) and malonate semialdehyde dehydrogenase (iolD). IolD is part of a possible operon, iolDEB, although the functions of IolE and IolB are unknown. The third locus, int, codes for an $\mathrm{ABC}$ transport system that is highly specific for myo-inositol. LacZ analysis showed that mutation of iolD, which codes for one of the last steps in the catabolic pathway, prevents increased transcription of the entire pathway. It is likely that the pathway is induced by an end product of catabolism rather than myo-inositol itself. Mutants in any of the loci nodulated peas (Pisum sativum) and vetch (Vicia sativa) at the same rate as the wild type. Acetylene reduction rates and plant dry weights also were the same in the mutants and wild type, indicating no defects in nitrogen fixation. When wild-type 3841 was coinoculated onto vetch plants with either catabolic mutant iolD (RU360) or iolA (RU361), however, $>95 \%$ of the nodules were solely infected with the wild type. The competitive advantage of the wild type was unaffected, even when the mutants were at 100-fold excess. The myo-inositol transport mutant (RU1487), which grows slowly on myo-inositol, was only slightly less competitive than the wild type. The nodulation advantage of the wild type was not the result of superior growth in the rhizosphere. Instead, it appears that the wild type may displace the mutants early on in the infection and nodulation process, suggesting an important role for myo-inositol catabolism.

Additional keywords: bacteria, competition.

myo-Inositol is abundant in pea and soybean nodules and is the most common compound inside isolated bacteroids of Bradyrhizobium japonicum (Sköt and Egsgaard 1984; Streeter 1987). The recent detection of inositol dehydrogenase activity, an enzyme induced specifically by inositol, in Rhizobium loti and Rhizobium fredii strains grown in solution extracted from a range of soils provides evidence that inositol also is present in soil and serves as an important substrate (Wood and

Corresponding author: P. S. Poole; Telephone: +44 118 9318895; Fax: +44 118 9318891; E-mail: p.s.poole@ reading.ac.uk
Stanway 2001). Given the presence of large amounts of myoinositol in legume nodules and soil, an important question about carbon flux concerns the regulation of myo-inositol utilization.

The importance of myo-inositol catabolism is illustrated by the synthesis of the myo-inositol derivatives known as rhizopines. For example, in alfalfa nodules, Sinohizobium meliloti strains L5-30 and Rm220-3 have been shown to produce rhizopines L-3-O-methyl-scyllo-inosamine (3-O-MSI) and scyllo-inosamine, respectively (Murphy et al. 1987; Murphy et al. 1988; Murphy et al. 1993; Saint et al. 1993). In strain L5-30, the genes for synthesis and catabolism of 3-OMSI are located on the nod-nif sym plasmid (Murphy et al. 1987; Murphy et al. 1988). Furthermore, the genes for synthesis of 3-O-MSI (but not its catabolism) are regulated symbiotically by nifA. It has therefore been hypothesized that rhizopines synthesized in the nodule by bacteroids may be catabolized by free-living bacteria in the rhizosphere. In pea nodules, the synthesis of ononitol and $O$-methyl-scylloinositol, which also belong to the inositol class of compounds, has been shown to depend on the strain of $R$. leguminosarum bv. viciae present, and strains of $R$. leguminosarum bv. viciae catabolize 3-O-MSI (Murphy and Saint 1992; Sköt and Egsgaard 1984; Wexler et al. 1995). The catabolism of rhizopines is dependent upon a functional myo-inositol catabolic pathway for $S$. meliloti and $R$. leguminosarum (Bahar et al. 1998; Galbraith et al. 1998).

The rhizopine-producing strain S. meliloti L5-30 had a competitive advantage for the nodulation of alfalfa (Medicago sativa) in soil when coinoculated with a mutant strain, even though when inoculated alone, the mutant had a similar rate of growth and nodulation to the wild type (Gordon et al. 1996). The mutant occupied less than $30 \%$ of nodules. This competitive advantage remained in soil 4 years after inoculation, even though there had been turnover of nodules in that time (Heinrich et al. 1999). Only approximately $10 \%$ of $S$. meliloti and R. leguminosarum strains, however, produce rhizopines (Wexler et al. 1995). Given that rhizopines must be catabolized via the myo-inositol catabolic pathway, we considered that myo-inositol also may be important in nodulation competition generally. There also is some evidence that disruption of myo-inositol dehydrogenase in S. meliloti may alter yields of alfalfa (Bosworth et al. 1994; Scupham et al. 1996). We therefore initiated a study to determine whether the ability to utilize myo-inositol is important for 
nodulation competitiveness in a strain of $R$. leguminosarum that does not make rhizopines.

\section{RESULTS}

Characterization of myo-inositol catabolic mutants.

The catabolic mutants RU360 and RU361 were identified previously by their inability to grow when myo-inositol was the sole carbon source (Poole et al. 1994a). The transposon insertions in these mutants were cloned, and the junction between Tn5 and strain 3841 DNA sequenced (Bahar et al. 1998). The transposons in strains RU360 and RU361 inserted into iolD and iolA, respectively. IolD is a putative acetolactate synthase, whereas IolA is a putative malonate semialdehyde dehydrogenase. These inositol mutants were then used to show that the rhizopine 3-O-methyl-scyllo-inosamine requires the inositol catabolic pathway for breakdown in R. leguminosarum.

Two cosmids, pRU3078 and pRU3079, which complement RU360 but not RU361, had been isolated (Poole et al. 1994a). This suggests that these mutations may be widely separated from each other in the genome of strain 3841. As part of our interest in the role of myo-inositol in rhizosphere growth and nodulation competition, we considered it essential to better define the nature of the insertions and operon structures. A cosmid library of strain 3841 DNA was introduced into strain RU361, and cosmid pRU3111, which complements growth, was isolated. pRU3111 did not complement strain RU360, however, which is consistent with the lack of complementation of strain RU361 by cosmids pRU3078 and pRU3079. All three cosmids also were Southern blotted with the Tn 5 clones from strains RU360 (pRU472) and RU361 (pRU476). As expected, pRU472 hybridized to pRU3078 and pRU3079 but not to pRU3111, whereas pRU476 hybridized only to pRU3111.

To fully define the mutation in strain RU360, the entire region was sequenced on both strands using plasmid pRU472 (Fig. 1). The region was then extended by direct sequencing of cosmid pRU3078. A total region of 5,606 bp was sequenced on both strands (EMBL accession no. AJ276296). BLASTX analysis of the deduced amino acid sequences revealed homology with protein IolDEB encoded by genes in the myoinositol degradation operon of Bacillus subtilis (Yoshida et al. 1997). A 9-bp repeat was created in RU360 by the insertion of Tn5-lacZ (1,775 to 1,784 bp of EMBL accession no. AJ276296, i.e., GGCAGCACT). The highest identity of the first gene in this putative operon was $41 \%$ ( $p$ value of $10^{-127}$ ) with IolD (acetolactate synthase) in the myo-inositol catabolic iol operon of B. subtilis. Downstream of iolD was an open reading frame (ORF) whose deduced amino acid sequence had homology with three proteins, MocC of R. leguminosarum $b v$. viciae, MocC of S. meliloti, and IolE of B. subtilis, in the databases. These proteins have no known function. The highest amino acid identity was $42 \%$ ( $p$ value of $3 \times 10^{-62}$ ) over the entire amino acid sequence of MocC of $R$. leguminosarum bv. viciae. The ORF was designated iolE and began $46 \mathrm{bp}$ downstream of the end of iolD. Downstream (246 bp) of the iolE
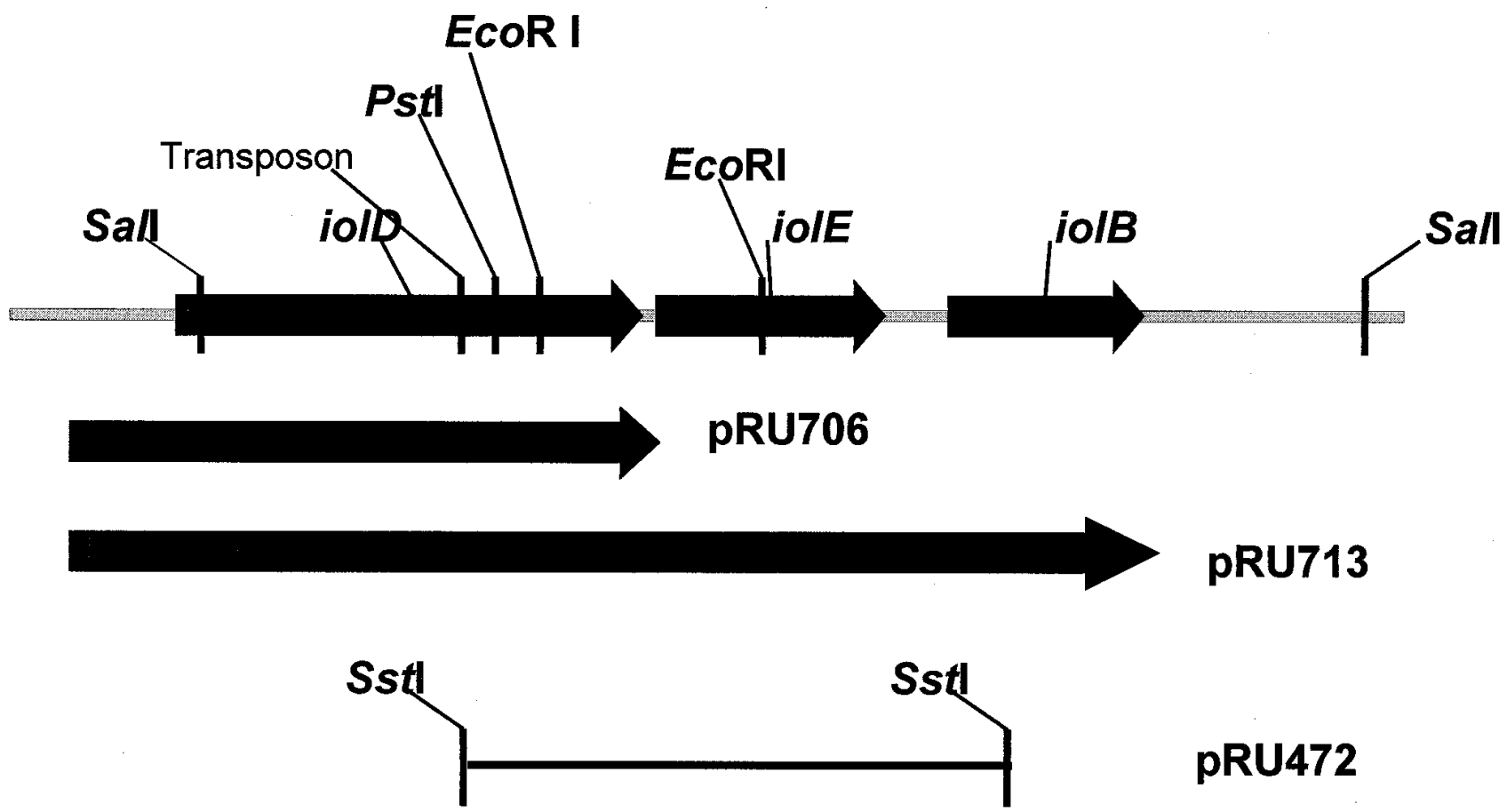

pRU713

pRU472

Fig. 1. iolDEB genes from Rhizobium leguminosarum 3841. Plasmid pRU472 was used for sequencing and is derived from strain RU360. SstI site is derived from Tn5-lacZ (left) and the chromosome of strain RU360 (right). 
gene was an ORF with $62 \%$ identity ( $p$ value of $5 \times 10^{-58}$ ) with IolB of B. subtilis. This protein has no other homologs in the databases and no known function. All three genes are predicted to be transcribed in the same direction as the $\beta$ galactosidase of Tn5-lacZ in strain RU360, but they are not organized in the same order as in B. subtilis. Putative ShineDalgarno sequences were identified for iolD (GAGGGG, 3 bp upstream) and iolB (AGGAGA, 8 bp upstream), but the only good match for iole (GGAGGA) was too far away (21 bp) from the putative ATG start.

The iolD gene is $1,881 \mathrm{bp}$ and encodes a predicted protein of 626 amino acids with a relative molecular mass of 66,730 and an isoelectric point of 6.1. The iolE gene is $939 \mathrm{bp}$ and encodes a predicted protein of 312 amino acids with a relative molecular mass of 34,456 and an isoelectric point of 5.6. The iolB gene is $795 \mathrm{bp}$ and encodes a predicted protein of 264 amino acids with a relative molecular mass of 29,046 and an isoelectric point of 5.6.

Further sequence was obtained for $1 \mathrm{~kb}$ on either side of the iol genes. A putative ORF that began $407 \mathrm{bp}$ downstream of iolB was identified. Sequencing did not extend far enough to give the end of the putative ORF. BLASTX analysis of the deduced amino acid sequence of the region surrounding the iol genes revealed no homology with known sequences. Sequencing of the region upstream of iolD did not reveal any homology with sequences in the databases. The sequence data suggest that the putative genes iolD, iolE, and iolB comprise an operon that is distinct from other genes involved in myoinositol utilization.

In order to determine whether all three genes identified in RU360 are necessary for myo-inositol catabolism, polymerase chain reaction (PCR) amplification was carried out on the iol region with cosmid pRU3078. Plasmids pRU706 (iolD) and pRU713 (iolDEB) were conjugated into RU360. Six colonies from each conjugation were streaked onto AMA medium containing $10 \mathrm{mM}$ myo-inositol as the sole carbon source. None of the colonies of strain RU360/pRU706 were able to grow on myo-inositol, indicating that the presence of the entire iolD gene was not sufficient to restore growth. All six colonies containing RU360/pRU713 grew on myo-inositol as the sole carbon source, indicating that the iolD, iolE, and iolB genes enable complementation. This suggests that the transposon insertion in iolD has a polar effect on the downstream genes (iolEB) and, therefore, one or both of these genes is essential for myo-inositol catabolism. It is consistent that iolDE and, perhaps, iolDEB are in an operon with a promoter upstream of iolD. The large intergenic region between iolE and iolB (246 bp) suggests that $i o l B$ may have its own promoter, although this was not investigated.

\section{Characterization of the iolA gene.}

An intact Tn5-lacZ with flanking chromosomal DNA was cloned previously from RU361 as an approximately 12,400-bp SalI fragment into pBluescript SK ${ }^{-}$(pRU438) (Bahar et al. 1998). The clone contained 230 bp of chromosomal DNA upstream of the transposon and approximately 3,800 bp downstream. Whereas the junctions of the transposon had been sequenced (Bahar et al. 1998), suggesting an insert in acetolactate synthase (iolA), the entire region needed to be sequenced fully. Therefore, a NotI subclone of pRU438 was constructed in pBluescript $\mathrm{SK}^{-}$(pRU476). Where appropriate, the sequence also was extended by direct sequencing from cosmid pRU3111, giving a complete sequence of 3,309 bp (EMBL accession no. AJ276297). A 9-bp repeat was created in RU361 by the insertion of Tn5-lacZ (1,525 to $1533 \mathrm{bp}$ in accession no. AJ276297, TAGCCTGCG). BLASTX analysis of the deduced amino acid sequence of the DNA revealed an ORF with homology to several methylmalonate semialdehyde dehydrogenases, including IolA of the B. subtilis iol operon. Highest identity was $68 \%$ with MmsA of Mycobacterium tuberculosis ( $p$ value of $1 \times 10^{-145}$ ). The ORF, designated iolA, is predicted to be transcribed in the opposite direction to the $\beta$-galactosidase in the transposon. The iolA gene is $1,497 \mathrm{bp}$ and has a putative Shine-Dalgarno sequence (AGGAGA) 6 bp upstream of the start site. It encodes a predicted protein of 498 amino acids and has a relative molecular mass of 53,639 and an isoelectric point of 5.8 .

Downstream of iolA, an ORF was identified whose deduced amino acid sequence had identity to Aau3 of $S$. meliloti (EMBL accession no. AF193764). The Aau3 protein is predicted to be required for growth on polyhydroxybutyrate cycle intermediates. There was $74 \%$ identity ( $p$ value of $6 \times 10^{-54}$ ) over the amino acid sequence. Other homologs included Yhde of B. subtilis and YjeB of Escherichia coli. None of the other homologs have any known function.

Sequencing was continued 1,206 bp upstream of the iolA gene with custom primers. An ORF was identified beginning at $405 \mathrm{bp}$ upstream of the start of iolA. BLASTX analysis of the deduced amino acid sequence showed that the ORF had $35 \%$ identity ( $p$ value of $9 \times 10^{-12}$ ) over the first 173 amino acids of 282 with a hypothetical gene from A. tumefaciens. The gene does not have any known function. The next highest homology was $31 \%$ ( $p$ value of $3 \times 10^{-9}$ ) with a hypothetical transcriptional activator gene, act, from Pseudomonas aeruginosa. The ORF, designated orfl, is predicted to be transcribed in the opposite direction to iolA. Sequencing was not extended further because our primary aim was to see whether any other genes involved in myo-inositol catabolism were likely to be present. The sequence clearly shows that iolA is isolated from other obvious ino genes. This is unlike the clustering of the entire ino operon in B. subtilis (Yoshida et al. 1997) but is consistent with the presence of the myo-inositol gene by itself in S. meliloti (Galbraith et al. 1998).

\section{LacZ fusion analysis of ino gene expression.}

In a previous study, we had shown that strains RU360 and RU361 did not induce the first two enzymes of the myoinositol catabolic pathway, myo-inositol dehydrogenase and 2-ketoinositol dehydratase (Poole et al. 1994a). The data in this study show that the failure to induce these first enzymes occurs in strains that are mutated in two of the last steps of the myo-inositol catabolic pathway. This suggests that induction of the pathway does not occur as the result of the presence of myo-inositol itself but may require elevated levels of later intermediates in the pathway. From the sequencing we conducted in this study, it is apparent that the Tn5-lacZ fusion in iolD (strain RU360) should be active. When $\beta$-galactosidase activity was originally measured in RU360, however, the fusion was inactive (Poole et al. 1994a). It was decided to repeat this assay and to test whether the fusion was active in RU360 when complemented by pRU3078 and pRU3079 (Table 1). Strain RU361 
was not tested because Tn5-lac Z is transcribed in the opposite direction to the interrupted gene.

The data show that $\beta$-galactosidase activity was very low in RU360 grown on $20 \mathrm{mM}$ pyruvate alone. The presence of 10 $\mathrm{mM}$ myo-inositol, in addition to pyruvate in the growth medium, did not cause the induction of expression of lacZ. When complemented with cosmids pRU3078 or pRU3079, however, there were two- and fivefold increases, respectively, in lac Z activity when myo-inositol was present, in addition to pyruvate. When RU360/pRU3078 and RU360/pRU3079 were grown on myo-inositol as the sole carbon source, there was an increase in the expression of lacZ of 12- and 20-fold, respectively. These data indicate that the genes interrupted by Tn5lacZ in RU360 are myo-inositol inducible but that a complete catabolic pathway is required for induction and that pyruvate partially represses induction.

\section{Isolation of a new inositol transport mutation.}

We show below that myo-inositol catabolic mutations (RU360 and RU361) are affected severely in their ability to compete for nodulation of peas and vetch. Because the effects of blocking catabolism can be varied, we conducted a new screen for $\operatorname{Tn} 5$ mutants in an attempt to isolate one that is blocked in myo-inositol uptake. Approximately 10,000 Tn5 mutants were screened on minimal medium with either glucose or myo-inositol as the sole carbon source. Of these, strain RU1487 was identified because it is severely impaired in its ability to grow on myo-inositol as the sole carbon source. Its growth rate when myo-inositol is the sole carbon source is almost four times slower than that of 3841 (mean generation time of $13.4 \mathrm{~h}$ for RU1487 and $3.4 \mathrm{~h}$ for 3841 ). To ascertain whether the reduced growth capability on myo-inositol also was the result of the insertion of $\mathrm{Tn} 5$, the transposon was transduced with the phage RL38 from RU1487 into 3841 by selecting for kanamycin resistance. Eleven transductants were tested for growth on AMA medium with either $10 \mathrm{mM}$ myoinositol or glucose as the sole carbon source. No growth was visible for any of the transductants on myo-inositol at least 8 days after they had been streaked out, whereas they all grew normally on glucose. This confirms the tight linkage of the transposon in strain RU1487 with the very poor ability to grow on myo-inositol.

An intact Tn5 was cloned from RU1487 into pBluescript $\mathrm{SK}^{-}$, but this proved unstable. Thus, a 7,900-bp EcoRI fragment with approximately $2,100 \mathrm{bp}$ of chromosomal DNA flanking both ends of the transposon was cloned into the lowcopy-number vector pACYC184 producing pRU426. The two ends of the transposon were PCR amplified with a Tn5 primer (p120) and primers that flank the EcoRI site of pACYC184 (p87 and p89). The PCR products were then sequenced with p113 (Tn5 specific primer) and either p114 or p115, which bind to the PCR products of p120/p87 and p120/p89, respectively. Sequencing was carried out over $2,100 \mathrm{bp}$, with a gap of approximately $400 \mathrm{bp}$ in the middle. A 9-bp overlap was created in RU1487 by the insertion of Tn5 (AGCTGAACC). BLASTX analysis of the deduced amino acid sequence revealed that the transposon interrupted an ORF with identity to the ATP binding component of ABC transport systems involved in the uptake of D-galactose and methyl-galactoside $(\mathrm{Mgl})$. The identity was $54 \%$ over the entire sequence of MglA of E. coli ( $p$ value of $2 \times 10^{-71}$ ). The gene encoded by the ORF was designated intA (myo-inositol transport). A putative Shine-Dalgarno sequence (AAGG) was identified 3 bp upstream of the ATG start. Ending 5 bp upstream of the putative start of the intA gene was an ORF whose deduced amino acid sequence had $75 \%$ identity ( $p$ value of $1 \times 10^{-38}$ ) over the final 100 amino acids of 309 of MocB of S. meliloti and $R$. leguminosarum bv. viciae. This gene encodes a periplasmic binding protein that binds to rhizopines and is thought to be part of a rhizopine transport system. The gene encoded by the ORF was designated int $B$ and is predicted to be transcribed in the same direction as intA. There also was high homology with D-galactose binding proteins, which also was the case for the myo-inositol binding protein of the Pseudomonas species (Deshusses and Belet 1984). On the basis of the sequence data, it can be postulated that strain RU1487 is mutated in an operon of genes encoding an $\mathrm{ABC}$ transport system that might be responsible for the uptake of myo-inositol or a derivative. The high identity of IntB with MocB (75\% identity to the last 100 amino acids of $\mathrm{MocB}$ ), also suggests the possibility that the int $\mathrm{ABC}$ uptake system may provide the membrane complex to which MocB binds to promote rhizopine uptake.

\section{Characterization of myo-inositol uptake.}

Various strains were grown on $20 \mathrm{mM}$ pyruvate or $20 \mathrm{mM}$ pyruvate plus $10 \mathrm{mM}$ myo-inositol as the sole carbon sources, and the uptake of myo-inositol was measured (Table 2). Uptake also was measured for 3841, and RU1487 when grown on $10 \mathrm{mM}$ myo-inositol alone. All uptake experiments were carried out with ${ }^{14} \mathrm{C}$-labeled glucose as a control.

There was a basal rate of myo-inositol uptake by each strain grown on $20 \mathrm{mM}$ pyruvate. This did not increase significantly in RU360 and RU1487 grown on pyruvate plus myo-inositol or RU1487 grown on myo-inositol alone. When strain 3841 was grown on $20 \mathrm{mM}$ pyruvate and $10 \mathrm{mM}$ myo-inositol or $10 \mathrm{mM}$ myo-inositol alone, however, there were 2.5- and sevenfold increases in the rate of uptake of myo-inositol, respectively, compared with the rate of uptake when grown on pyruvate. These data indicate that there is an inducible system for the transport of myo-inositol in 3841 but not in the mutants. There was no significant difference in the rate of glucose uptake by the strains on different carbon sources (data not shown).

Table 1. $\beta$-Galactosidase activity of RU360 ${ }^{\mathrm{a}}$

\begin{tabular}{lccc}
\hline & \multicolumn{2}{c}{$\beta$-Galactosidase activity/nmol/min/mg of protein grown on different carbon sources } \\
\cline { 2 - 4 } Strain & Pyruvate & Pyruvate $+\boldsymbol{m y o}$-inositol & myo-Inositol \\
\hline RU360 & $160.3 \pm 15.5$ & $132.2 \pm 4.1$ & - \\
RU360/pRU3078 & 125.7 & 264.8 & $1,453.6$ \\
RU360/pRU3079 & $90.0 \pm 4.3$ & $459.4 \pm 35.3$ & $1,871.4 \pm 315.9$ \\
\hline
\end{tabular}

${ }^{a}$ Each value represents the mean of three cultures with standard deviation. RU360/pRU3078 is the mean of two cultures. RU360 cannot be grown on myo-inositol as the sole carbon source. 
The deduced amino acid sequence of the putative intA gene of RU1487 has high homology to MglA, a component of the galactose and Mgl uptake system. Therefore, the uptake system might not be specific for myo-inositol. To determine the specificity of the transport system, myo-inositol uptake by 3841 was measured after the addition of a fivefold excess of different carbon compounds (Table 2). The inducible system is

Table 2. Transport of myo-inositol ${ }^{\mathrm{a}}$

\begin{tabular}{lllc}
\hline & \multicolumn{2}{c}{ Transport rate/nmol/min/mg of protein } \\
\cline { 2 - 4 } Strain & \multicolumn{1}{c}{ Grown on } & Competitor & Transport \\
\hline 3841 & Pyruvate & - & $6.4 \pm 1.4$ \\
3841 & Pyruvate/inositol & - & $15.1 \pm 3.5$ \\
3841 & Inositol & - & $47.0 \pm 1.7$ \\
3841 & Inositol & Mannitol & $37 \pm 5.6$ \\
3841 & Inositol & Sorbitol & $35.2 \pm 12.0$ \\
3841 & Inositol & Glucose & $41.6 \pm 2.9$ \\
3841 & Inositol & Fructose & $36.0 \pm 5.8$ \\
3841 & Inositol & Pyruvate & $40.2 \pm 10.7$ \\
RU1487 & Pyruvate & - & $5.9 \pm 0.8$ \\
RU1487 & Pyruvate/inositol & - & $4.7 \pm 1.3$ \\
RU1487 & Inositol & - & $7.4 \pm 1.5$ \\
RU360 & Pyruvate & - & $5.9 \pm 0.2$ \\
RU360 & Pyruvate/inositol & - & $5.7 \pm 1.5$ \\
RU361 & Pyruvate & - & $5.4 \pm 0.2$ \\
RU361 & Pyruvate/inositol & - & $9.2 \pm 1.6$ \\
\hline
\end{tabular}

a Transport rates are shown as the mean of three independent cultures \pm standard deviation. myo-Inositol was at $25 \mu \mathrm{M}$, and competitors were at a fivefold excess. All cultures were grown on acid minimal salts minimal medium, with carbon sources indicated. clearly specific for myo-inositol. The presence of a basal background rate of uptake, presumably the result of a second transport system, explains the poor growth of RU1487 on myo-inositol. It also is notable that the absence of myo-inositol transport elevation in strains RU360 or RU361 grown on pyruvate/myo-inositol indicates an absence of induction, unless there is a complete catabolic pathway. This is the same pattern as observed with LacZ fusion analysis of iolD (Table 1).

\section{Nodulation ability of the mutants.}

Strains RU360, RU361, and RU1487 were able to nodulate vetch at the same rate as 3841 , with the same numbers of nodules formed when $10^{3}$ cells were applied to the roots of 7day-old seedlings (Fig. 2). Low inocula were used because high levels of inocula can bias competition experiments, particularly where there are growth rate differences between strains (Streit et al. 1996). Strain RU360 was shown previously to reduce acetylene at the same rate as 3841 on vetch (Poole et al. 1994a). Strains 3841, RU361, and RU1487 reduced acetylene on pea at $2.4 \mu$ mole per h per plant (standard error of the mean [SEM] 0.45), $2.4 \mu$ mole per $\mathrm{h}$ per plant (SEM 0.1), and $2.9 \mu$ mole per h per plant (SEM 0.7), respectively, indicating that these strains are not affected in their ability to fix nitrogen. The strains also were inoculated onto common vetch (Vicia sativa), which is a small seeded legume that shows severe nitrogen deficiency if nitrogen fixation is impaired. Average plant dry weights for vetch inoculated with 3841, RU361, and RU1487 were 0.64 (SEM 0.14), 0.65 (SEM 0.19), and $0.78 \mathrm{~g}$ (SEM 0.13), respectively, indicating no significant effects.

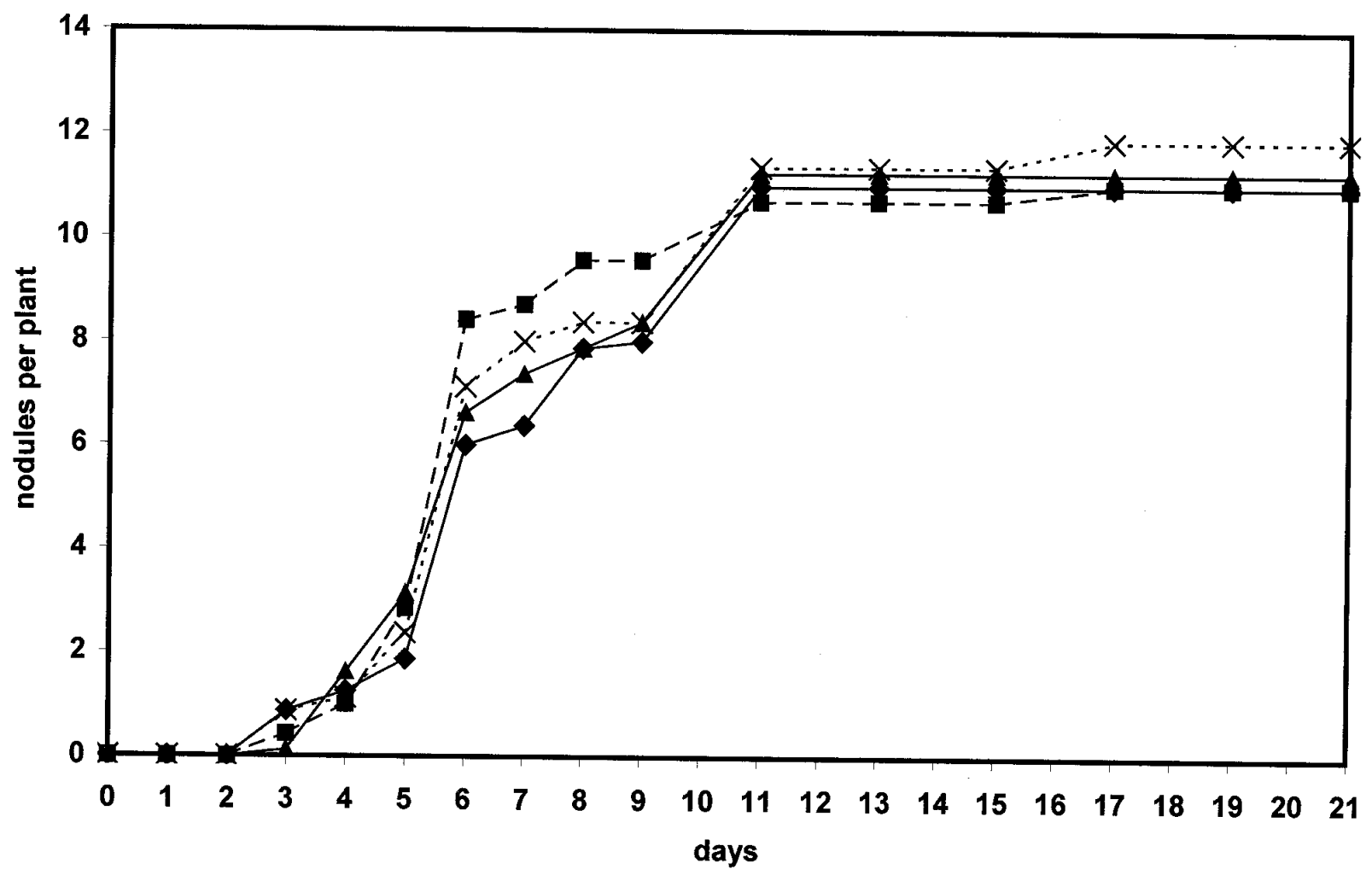

Fig. 2. Nodulation kinetics of vetch by several strains of Rhizobium. leguminosarum. $\times, 3841$; $\mathbf{\Delta}$, RU1487; $\mathbf{\square}$, RU361; and $\bullet$, RU360. Each strain $\left(10^{3}\right.$ CFU) was inoculated onto eight plants. Analysis of variance showed that none of the differences are statistically different. 
Nodulation competitiveness of the mutants.

The above data as well as the previous demonstration that the first two enzymes of myo-inositol catabolism are not induced in mature pea bacteroids demonstrates that myo-inositol is not important as a carbon source to fuel nitrogen fixation in either pea or vetch. This is hardly surprising given the importance of dicarboxylic acids in this process (Poole and Allaway 2000). The abundance of myo-inositol in legume nodules as well as the rhizosphere and the role of the myo-inositol catabolic pathway in rhizopine degradation, however, suggests it may be important in earlier steps in growth in the rhizosphere or in nodulation (Bahar et al. 1998; Galbraith et al. 1998; Sköt and Egsgaard, 1984; Streeter 1987; Wood and Stanway 2001). We therefore examined the effect of myo-inositol catabolic pathway mutation on growth in the rhizosphere and in competitiveness for nodulation.

When inoculated alone, 3841, RU360, RU361, and RU1487 were the sole occupants of all nodules, as appropriate (Table $3)$. No nodules were obtained on plants inoculated with sterile acid minimal salts (AMS) alone. When 3841 and RU360 were coinoculated onto vetch in equal numbers $\left(10^{3} \mathrm{CFU}\right), 3841$ occupied $97.6 \%$ of nodules as the sole occupant. Strain 3841 maintained dominance when RU360 was inoculated in tenfold and 100-excess, with 3841 the sole occupant in 99.4 and 95.9\% of nodules, respectively (Table 3 ). When 3841 and RU361 were coinoculated onto vetch in equal numbers $\left(10^{3}\right.$ CFU), 3841 occupied $100 \%$ of nodules as sole occupant. Strain 3841 occupied 89.5 and $98 \%$ of nodules as sole occupant when RU361 was in ten- and 100-fold excess, respectively (Table 3).

Even though very few nodules containing any kanamycinresistant bacteria were obtained, the possibility of coresidence in nodules was checked in 12 of these rare nodules from plants, which had been inoculated with equal numbers $\left(10^{3}\right)$ of either RU360/3841 or RU361/3841 (72 colonies for each combination). For those plants inoculated with RU360/3841, seven of the 12 nodules had a mixture of kanamycin-resistant (RU360) and -sensitive bacteria (3841). Strain 3841 made up $9 \%$ of the colonies isolated from the mixed occupancy nodules. For those plants inoculated with RU361/3841, nine out of 12 nodules had a mixture of kanamycin-resistant (RU361) and -sensitive bacteria (3841). Strain
3841 made up $19 \%$ of the colonies isolated from the mixed occupancy nodules. The mutants predominate in these nodules but the wild type is still present in a majority of mixedoccupancy nodules. This contrasts with the vast majority of nodules that were occupied solely by 3841 . RU360 and RU361 containing complementing cosmids were competed with the wild type, however, whereas the number of nodules containing a mutant increased dramatically, although the cosmid retention was too low to draw firm conclusions (data not shown). Bacteria isolated from control plants inoculated only with mutants had $100 \%$ retention of the kanamycin marker, demonstrating that the presence of kanamycin-sensitive wildtype bacteria in coinoculated plants is not the result of reversion.

The same trends were observed for pea coinoculated with 3841 and RU360 or RU361 (data not shown). These data demonstrate a profound competitive advantage for strains able to catabolize myo-inositol. Whereas any single mutation may cause a loss of competition for allele-specific reasons, these mutations are in genes that are not part of the same operon and encode proteins for separate reactions. This makes it highly likely that myo-inositol catabolism, per se, is important in nodulation competitiveness. Disrupting a large metabolic pathway such as that for myo-inositol, however, may have a range of effects on metabolism, so we considered it important to isolate a transport mutant (see above) and test its effects on nodulation competitiveness.

When vetch plants were coinoculated with RU1487 and 3841 in equal numbers $\left(10^{3} \mathrm{CFU}\right), 3841$ was the sole occupant in $52.9 \%$ of the nodules. When RU1487 was inoculated in tenand 100-fold excess, 3841 was the sole occupant in 30.7 and $19.1 \%$ of the nodules, respectively (Table 3). Co-residence was assessed in 12 nodules that contained kanamycin-resistant bacteria. Nine of the twelve nodules had a mixture of kanamycin-resistant (RU1487) and -sensitive bacteria (3841), whereas three contained RU1487 alone. Strain 3841 made up $19 \%$ of the bacteria. Overall, the data indicate that with equal inocula, strain 3841 is present as sole occupant in 53\% of nodules, RU1487 is the sole occupant in approximately $12 \%$ of nodules, and $35 \%$ of nodules have at least some coresidence. This indicates a slight competitive advantage for 3841 over RU1487, a result supported by the fact that a 100-

Table 3. Competition for nodule occupancy ${ }^{\mathrm{a}}$

\begin{tabular}{|c|c|c|c|c|c|c|}
\hline \multirow[b]{2}{*}{ Strains } & \multirow[b]{2}{*}{ Inoculum Ratio } & \multicolumn{5}{|c|}{ Number of nodules } \\
\hline & & Total & 3841 & RU360 & RU361 & RU1487 \\
\hline None & & 0 & & & & \\
\hline 3841 & & 72 & 72 & & & \\
\hline RU360 & & 72 & & 72 & & \\
\hline RU361 & & 72 & & & 72 & \\
\hline RU1487 & & 72 & & & & 72 \\
\hline RU360/3841 & $1: 1$ & 84 & 82 & 2 & & \\
\hline RU360/3841 & $10: 1$ & 176 & 175 & 1 & & \\
\hline RU360/3841 & 100:1 & 194 & 186 & 8 & & \\
\hline RU361/3841 & $1: 1$ & 116 & 116 & & 0 & \\
\hline RU361/3841 & $10: 1$ & 191 & 171 & & 20 & \\
\hline RU361/3841 & $100: 1$ & 159 & 156 & & 3 & \\
\hline RU1487/3841 & $1: 1$ & 172 & 91 & & & 81 \\
\hline RU1487/3841 & $10: 1$ & 189 & 58 & & & 131 \\
\hline RU1487/3841 & $100: 1$ & 183 & 35 & & & 148 \\
\hline
\end{tabular}

${ }^{a}$ A $1 \times$ inoculum was $10^{3} \mathrm{CFU}$ on vetch plants. Nodules scored as containing the wild-type bacteria did not contain any detectable mutants, although nodules containing mutant strains often contained wild type as well (see text). 
fold excess of RU1487 reduces sole 3841 occupancy only to $19.1 \%$. This is dramatically different from the myo-inositol catabolic mutants RU360 and RU361, which show almost complete dominance by the wild type, even when the mutants are inoculated in 100-fold excess.

The growth of RU360, RU361, RU1487, and 3841 in the rhizosphere of vetch was monitored at 2, 4, 6, and 8 days, immediately following inoculation. Analysis of variance showed that there were no significant differences in the final numbers of any strains $(P>0.05)$. Thus, differences in nodule occupancy are very unlikely to be the result of different growth rates in the rhizosphere.

We also considered the possibility that the presence of myoinositol itself may be toxic to ino mutants. Such a possibility might explain the nodulation competition difference between the transport mutant RU1487 and the catabolic mutants RU360 and RU361. Strains 3841, RU1487, RU360, and RU361, however, grew with mean generation times of 4.1, $4.1,3.9$, and $3.9 \mathrm{~h}$, respectively, on myo-inositol plus pyruvate minimal medium.

\section{Wild type apparently displaces the mutant during development.}

The effect of different inoculum levels on the total number of nodules formed on vetch was investigated (Fig 3). There were no significant differences between the number of nodules formed by 3841, RU1487, RU360, or RU361 when inoculated $\left(10^{3} \mathrm{CFU}\right)$ alone. When a 10 -fold excess of mutant was added $\left(10^{4} \mathrm{CFU}\right)$ with wild type $\left(10^{3} \mathrm{CFU}\right)$, there was a statistically significant $(P<0.1)$ increase in the total number of nodules formed. In the case of RU360 and RU361 excess, this result is remarkable because the increased nodulation is in response to the presence of mutant bacteria that occupy few of the mature nodules. These results suggest that the plant is able to detect the presence of extra bacteria in the rhizosphere, perhaps as a result of elevated levels of lipochitooligosaccharides, which then elicit greater numbers of nodules. The absence of the mutants in mature nodules suggests that they have been displaced by the wild type at an early stage of nodule formation or during infection thread formation. It seems relevant that in all situations where mutants of RU360, RU361, or even RU1487 were recovered from nodules, there also was a high incidence of co-resident wild-type bacteria.

\section{DISCUSSION}

The data presented here indicate that mutants RU360, RU361, and RU1487 are able to nodulate pea and vetch at the same rate as 3841 . Bacteroids formed by all the mutants fix nitrogen at the same rate, and the average plant dry weights are the same. The myo-inositol catabolic mutants RU360 and RU361, however, suffer a severe disadvantage in competition for nodulation when coinoculated with 3841. Strain RU1487, which is not a catabolic myo-inositol mutant, retains the ability to catabolize myo-inositol at a low rate and is only at a very slight competitive disadvantage when coinoculated with 3841. This suggests that there is a requirement to utilize myoinositol in order to be competitive for nodulation. A key question is, at what stage does this competitive advantage manifest itself? The ino mutants RU360 and RU361 grew as fast as the

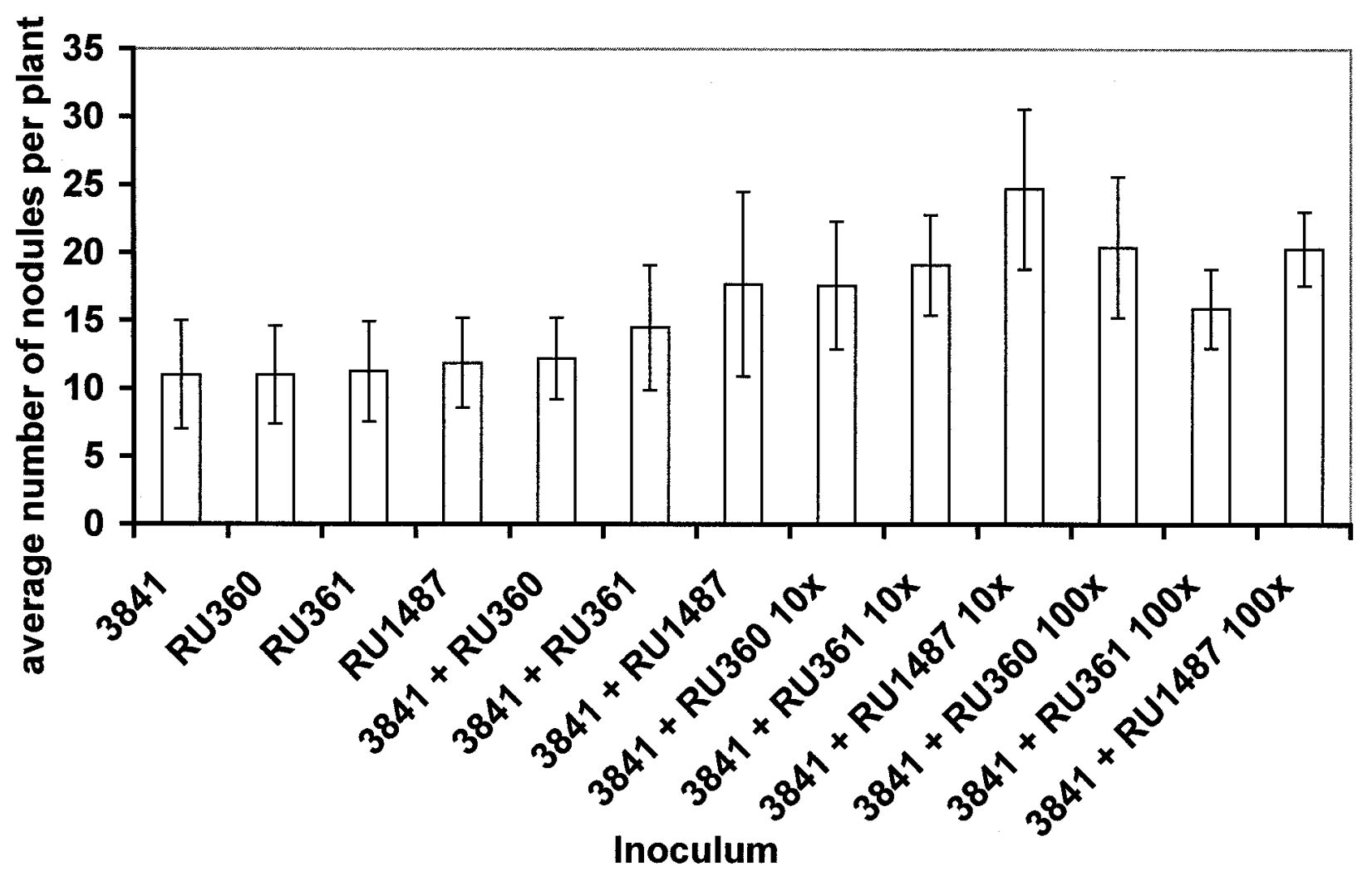

Fig. 3. Effect of inoculation level on nodulation frequency of vetch. Standard bacterial inoculum was $10^{3} \mathrm{CFU}$. Higher inocula are indicated as $10 \times\left(10^{4}\right.$ $\mathrm{CFU})$ and $100 \times\left(10^{5} \mathrm{CFU}\right)$. A minimum of six plants were harvested for each treatment. Results are shown plus and minus the standard deviation. 
wild type in the rhizosphere and elicited nodule formation at a similar rate to that of the wild type (Fig 2). Plant nodulation also responded to the size of the mutant inoculum in the presence of wild type, suggesting that there is no problem with lipochitooligosaccharide generation. What was notable, however, was that whereas the total number of nodules increased 10:1 ratio of mutant to wild type, the nodules contained wildtype bacteria and not the mutant. This indicates that the ino mutants are not competitive with the wild type during the early stages of nodule development and infection thread formation. This possibility also is supported by a high incidence of co-residence of the wild type with the mutant in any nodules that contained mutant bacteria. The apparent ability of the wild type to displace the mutant suggests that the wild type may grow slightly faster at a critical stage such as during infection thread development or, perhaps, at a particular developmental stage. One possibility that seems unlikely is that myo-inositol is the sole carbon for growth in the early stages of infection. This is because the transport mutant RU1487 is severely impaired for growth on myo-inositol (MGT $13.4 \mathrm{~h}$ compared with $3.4 \mathrm{~h}$ for the wild type), yet it is still moderately competitive. Overall, the poor growth of RU1487 on myo-inositol is compatible with its slight reduction in competitiveness, supporting the idea that myo-inositol has a real role in the early stages of nodule development and that the lack of competitiveness of the catabolic mutants is not simply the result of myo-inositol toxicity.

Mutants of $R$. leguminosarum bv. trifolii that are unable to catabolize rhamnose are at a severe disadvantage for clover nodulation, although this did not occur in sorbitol or adonitol mutants (Oresnik et al. 1998). Whereas the effect of rhamnose and myo-inositol may be specific for each biovar of $R$. leguminosarum and plant host, it is possible that multiple carbon sources might affect competition. For example, several different compounds may contribute to the growth rates of strains.
One simple possibility is that infection threads containing myo-inositol mutants become aborted, leading to a dominance of the wild type. Certainly, many more infection threads are formed than lead to nodule formation (Bauer 1981). The increased nodulation response of vetch plants when an excess of mutants are added with the wild type, however, indicates that the effects seen here are not so simple. The abortion of infection threads containing the mutant alone should not lead to more nodules; instead, the wild type appears to displace the mutant. In many ways, this study and that of Oresnik et al. (1998) highlights our lack of knowledge concerning early events in nodulation other than those concerned directly with flavonoid detection and lipochitooligosaccharide synthesis.

\section{MATERIALS AND METHODS}

\section{Bacterial strains and culture conditions.}

Bacterial strains and plasmids used in this study are described in Table 4. R. leguminosarum strains were grown at $28^{\circ} \mathrm{C}$ on either TY (Beringer 1974) or AMS medium (pH 7.2) (Poole et al. 1994b). All carbon and nitrogen sources were at $10 \mathrm{mM}$ concentrations unless otherwise stated. Antibiotics were used at the following concentrations (per ml): $40 \mu \mathrm{g}$ of kanamycin; $500 \mu \mathrm{g}$ of streptomycin; $2 \mu \mathrm{g}$ of tetracycline in AMS medium and $5 \mu \mathrm{g}$ in TY medium; and $20 \mu \mathrm{g}$ of gentamicin. E. coli strains were grown at $37^{\circ} \mathrm{C}$ on Luria-Bertani medium, with antibiotic concentrations as follows (per ml): 25 $\mu \mathrm{g}$ of kanamycin; $10 \mu \mathrm{g}$ of tetracycline; $5 \mu \mathrm{g}$ of gentamicin; and $50 \mu \mathrm{g}$ of ampicillin.

\section{DNA and genetic manipulations.}

All routine DNA analysis was performed essentially as described by Sambrook et al. (1989). Transduction was performed with bacteriophage RL38, as described by BuchananWollaston (1979). Tn5 was transduced from RU1487 into

Table 4. Bacterial strains and plasmids used

\begin{tabular}{|c|c|c|}
\hline Strain or plasmid & Description & Source or reference \\
\hline \multicolumn{3}{|c|}{ Rhizobium leguminosarum strains } \\
\hline 3841 & $\operatorname{Str}^{\mathrm{r}}$ derivative of $R$. leguminosarum bv. viciae strain 300 & Glenn et al. 1980 \\
\hline RU360 & Strain 3841 iolD::Tn5-lacZ & Poole et al. 1994a \\
\hline RU361 & Strain 3841 iolA::Tn5-lacZ & Poole et al. 1994a \\
\hline RU1487 & Strain 3841 int $A:: \operatorname{Tn} 5$ & This work \\
\hline \multicolumn{3}{|l|}{ Plasmids } \\
\hline pACYC184 & $\begin{array}{l}\text { Low-copy-number cloning vector; chloramphenicol and tetracy- } \\
\text { cline resistant }\end{array}$ & New England Biolabs Beverly, MA, U.S.A. \\
\hline pBluescript $\mathrm{SK}^{-}$ & $\begin{array}{l}\text { Phagemid; } \mathrm{F}_{1}^{-} \text {origin of replication; ColE1 replicon; ampicillin } \\
\text { resistant. }\end{array}$ & Stratagene, La Jolla, CA, U.S.A. \\
\hline PCR2.1 TOPO & T-overhang cloning vector; ampicillin and kanamycin resistant & Invitrogen, San Diego, CA, U.S.A. \\
\hline pRU426 & 12.4-kb EcoRI fragment from RU1427 (intA) in pACYC184 & This work \\
\hline pRU438 & $\begin{array}{l}\text { 11.5-kb Sall fragment containing all of Tn5-lacZ and part of iolA } \\
\text { from RU361 in pBluescript SK }\end{array}$ & This work \\
\hline pRU472 & $\begin{array}{l}\text { 9-kb SstI fragment containing } 6 \mathrm{~kb} \text { of } \mathrm{Tn} 5-\text {-lacZ from RU360 in } \\
\text { pBluescript } \mathrm{SK}^{-}\end{array}$ & This work \\
\hline pRU476 & $\begin{array}{l}\text { 1.2-kb NotI fragment containing IS } 50 R \text { of Tn5-lacZ and part of } \\
\text { iolA from pRU438 in pBluescript } \mathrm{SK}^{-}\end{array}$ & This work \\
\hline pRU706 & $\begin{array}{l}\text { 2.5-kb polymerase chain reaction (PCR) product of iolD (primers } \\
\text { p213 and p228) from pRU3078 cloned in pOT1 }\end{array}$ & This work \\
\hline pRU713 & $\begin{array}{l}\text { 4-kb PCR product of iolDEB (primers p230 and p228) from } \\
\text { pRU3078 cloned in pOT1 }\end{array}$ & This work \\
\hline pRU3078 & pLAFR1 cosmid that complements RU360; tetracycline resistant & Poole et al. 1994a \\
\hline pRU3079 & Cosmid that overlaps pRU3078 and complements RU360 & Poole et al. $1994 \mathrm{a}$ \\
\hline pRU3111 & pLAFR1 cosmid that complements RU361; tetracycline resistant & This work \\
\hline
\end{tabular}


3841. Eleven kanamycin-resistant transductants were tested for growth on AMA medium with $10 \mathrm{mM}$ myo-inositol as the sole carbon source. Chromosomal DNA from strain RU1487 was digested with EcoRI, and a 7.9-kb fragment was cloned into Bluescript II $\mathrm{SK}^{-}$by selecting for kanamycin resistance. The left and right arms of transposon $\mathrm{Tn} 5$ were isolated by BamHI and HindIII digestion, followed by self-ligation. Both arms were sequenced with a primer that binds to the ends of IS50 and SK and KS primers in the plasmid.

Primers p213 and p228 were used to amplify a 2,308-bp fragment containing the entire iolD gene. Primer p228 contains a SpeI site. This fragment was then cloned into plasmid pCR2.1 TOPO and then into pOT1 as an XbaI-SpeI fragment, generating pRU706. Primers p230 and p216 were used to amplify a 4,343-bp fragment containing the iolDEB genes, ending $38 \mathrm{bp}$ beyond the end of iolB. This fragment was cloned into pCR2.1 TOPO and then into pOT1 with PmeI and SpeI restriction enzyme sites that were included in p230 and p216, respectively. The resulting plasmid was named pRU713.

Primers used in this study were p87, TTAAATCAAAACTGGTGAAACTC; p89, GCTAAAATGGAGAAAAAAATCACTGG; p113, AGGTCACATGGAAGTCAGATC; p114, ACGTTTCAGTTTGCTCATGG; p115, AAAGACCGTAAAGAAAAATAAGC; p120, TTGATTTACCAGAATATTTTGCC; p213, TTTTTTTTCTGCAGCGAGCTGATTTCCTGCTTCG; p216, AAAAAAAAACTAGTTTCGCGCTGTCAGATTATTT; p228, TTTTTTTTACTAGTGCGGAGGAGCGGTGCCG; p230, TTTTTTTTGTTTAAACCGAGCTGATTTCCTGCTTCG.

All sequencing was performed by the cycle-sequencing method with an ALF automated DNA sequencer (Amersham Pharmacia Biotech, Little Chalfont, Buckinghamshire, U.K.). Genetics Computer Group software (Madison, WI, U.S.A.) and BLASTX analysis were used for computer-assisted sequence analysis. The EMBL accession nos. for iolA and iolDEB are AJ276297 and AJ276296, respectively.

\section{Plant assays.}

Nodulation and acetylene reduction were determined with common vetch (V. sativa) or pea (Pisum sativum) plants. Plant growth and acetylene reductions were carried out as described previously (Poole et al. 1994a). For nodulation competition experiments, plants were grown in sterile vermiculite and watered with sterile nitrogen-free rooting solution, as described previously (Poole et al. 1994a). An inoculum of $10^{3}$, $10^{4}, 10^{5}$, or $10^{6} \mathrm{CFU}$ was applied to each plant. Cell numbers were ascertained by a measurement of optical density (OD) at $600 \mathrm{~nm}$ and confirmed by plate counts. Plants were harvested 6 weeks postinoculation. To determine nodule occupancy, nodules were surface sterilized, as previously described (Poole et al. 1994a), then crushed and plated on TY medium and then on TY medium containing kanamycin. A minimum of six plants were harvested for each treatment. Samples also were purified from the original TY medium plates to check the resistance marker in individual bacteria and to determine their ability to grow on myo-inositol as the sole carbon source. Total bacterial growth in the rhizosphere was determined by harvesting plants at 2, 4, 6, and 8 days. Roots and total vermiculite were ground, and bacteria was serially diluted and plate counted on TY medium, giving the total number of viable rhizosphere and root-associated bacteria.

\section{Transport assays.}

Cells were prepared for transport assays, as previously described (Poole et al. 1985). The total myo-inositol concentration was $25 \mu \mathrm{M}$, with $0.125 \mu \mathrm{Ci}$ of $\mathrm{D}\left[\mathrm{U}_{-}{ }^{14} \mathrm{C}\right]$ myo-inositol $(310$ $\mathrm{MBq}$ per mmol) added. Samples of $0.1 \mathrm{ml}$ were taken at intervals of $1 \mathrm{~min}$, for up to $5 \mathrm{~min}$. Millipore was filtered and scintillation counted. For competition assays, a fivefold excess $(125 \mu \mathrm{M})$ of unlabeled solute was added $5 \mathrm{~s}$ prior to the addition of the myo-inositol.

\section{ACKNOWLEDGMENTS}

We thank the Biotechnology and Biological Sciences Research Council U.K. as well as the University of Reading for supporting this research.

\section{LITERATURE CITED}

Bahar, M., De Majnik, J., Wexler, M., Fry, J., Poole, P. S., and Murphy, P. J. 1998. A model for the catabolism of rhizopine in Rhizobium leguminosarum involves a ferredoxin oxygenase complex and the inositol degradative pathway. Mol. Plant-Microbe Interact. 11:10571068 .

Bauer, W. D. 1981. Infection of legumes by rhizobia. Annu. Rev. Plant Physiol. 32:407-449.

Beringer, J. E. 1974. R factor transfer in Rhizobium leguminosarum. J. Gen. Microbiol. 84:188-198.

Bosworth, A. H., Williams, M. K., Albrecht, K. A., Kwiatkowski, R., Beynon, J., Hankinson, T. R., Ronson, C. W., Cannon, F., Wacek, T. J., and Triplett, E. W. 1994. Alfalfa yield response to inoculation with recombinant strains of Rhizobium meliloti with an extra copy of $d c t A B D$ and/or modified nifA expression. Appl. Environ. Microbiol. 60:3815-3832.

Buchanan-Wollaston, V. 1979. Generalized transduction in Rhizobium leguminosarum. J. Gen. Microbiol. 112:135-142.

Dehusses, J., and Belet, M. 1984. Purification and properties of the myoinositol-binding protein from a Pseudomonas sp. J. Bacteriol. 159:179-183.

Galbraith, M. P., Feng, S. F., Borneman, J., Triplett, E. W., De Bruijn, F. J., and Rossbach, S. 1998. A functional myo-inositol catabolism pathway is essential for rhizopine utilization by Sinorhizobium meliloti. Microbiology (U.K.) 144:2915-2924.

Glenn, A. R., Poole, P. S., and Hudman, J. F. 1980. Succinate uptake by free-living and bacteroid forms of Rhizobium leguminosarum. J. Gen. Microbiol. 119:267-271.

Gordon, D. M., Ryder, M. H., Heinrich, K., and Murphy, P. J. 1996. An experimental test of the rhizopine concept in Rhizobium meliloti. Appl. Environ. Microbiol. 62:3991-3996.

Heinrich, K., Gordon, D. M., Ryder, M. H., and Murphy, P. J. 1999. A rhizopine strain of Sinorhizobium meliloti remains at a competitive nodulation advantage after an extended period in the soil. Soil Biol. Biochem. 31:1063-1065.

Murphy, P. J., and Saint, C. P. 1992. Rhizopines in the legumeRhizobium symbiosis. Pages 377-390 in: Molecular Signals in PlantMicrobe Communications. D. P. S. Verma, ed. CRC Press, Boca Raton, FL, U.S.A.

Murphy, P. J., Heycke, N., Banfalvi, Z., Tate, M. E., Debruijn, F., Kondorosi, A., Tempe, J., and Schell, J. 1987. Genes for the catabolism and synthesis of an opine-like compound in Rhizobium meliloti are closely linked and on the Sym plasmid. Proc. Natl. Acad. Sci. USA 84:493-497.

Murphy, P. J., Heycke, N., Trenz, S. P., Ratet, P., De Bruijn, F., and Schell, J. 1988. Synthesis of an opine-like compound, a rhizopine, in alfalfa nodules is symbiotically regulated. Proc. Natl. Acad. Sci. USA 85:9133-9137.

Murphy, P. J., Trenz, S. P., Grzemski, W., De Bruijn, F. J., and Schell, J. 1993. The Rhizobium meliloti rhizopine mos locus is a mosaic structure facilitating its symbiotic regulation. J. Bacteriol. 175:5193-5204.

Oresnik, I. J., Pacarynuk, L. A., O'Brien, S. A. P., Yost, C. K., and Hynes, M. F. 1998. Plasmid-encoded catabolic genes in Rhizobium leguminosarum bv. trifolii: Evidence for a plant-inducible rhamnose 
locus involved in competition for nodulation. Mol. Plant-Microbe Interact. 11:1175-1185.

Poole, P. S., and Allaway, D. A. 2000. Carbon and nitrogen metabolism in Rhizobium. Adv. Microb. Physiol. 43:117-163.

Poole, P. S., Franklin, M., Glenn, A. R., and Dilworth, M. J. 1985. The transport of L-glutamate by Rhizobium leguminosarum involves a common amino acid carrier. J. Gen. Microbiol. 131:1441-1448.

Poole, P. S., Blyth, A., Reid, C. J., and Walters, K. 1994a. myo-Inositol catabolism and catabolite regulation in Rhizobium leguminosarum bv. viciae. Microbiology 140:2787-2795.

Poole, P. S., Schofield, N. A., Reid, C. J., Drew, E. M., and Walshaw, D. L. 1994b. Identification of chromosomal genes located downstream of $d c t D$ that affect the requirement for calcium and the lipopolysaccharide layer of Rhizobium leguminosarum. Microbiology 140:2797-2809.

Saint, C. P., Wexler, M., Murphy, P. J., Tempe, J. and Tate, M. E. 1993. Characterization of genes for synthesis and catabolism of a new rhizopine induced in nodules by Rhizobium meliloti Rm220-3: Extension of the rhizopine concept. J. Bacteriol. 175:5205-5215.

Sambrook, J., Fritsch, E. F., and Maniatis, T. 1989. Molecular Cloning: A Laboratory Manual, 2nd ed. Cold Spring Harbor Laboratory, Cold Spring Harbor, NY, U.S.A

Scupham, A. J., Bosworth, A. H., Ellis, W. R., Wacek, T. J., Albrecht, K.
A., and Triplett, E. W. 1996. Inoculation with Sinorhizobium meliloti rmbpc-2 increases alfalfa yield compared with inoculation with a nonengineered wild-type strain. Appl. Environ. Microbiol. 62:42604262 .

Sköt, L., and Egsgaard, H. 1984. Identification of ononitol and Omethyl-scyllo-inositol in pea root nodules. Planta 161:32-36.

Streeter, J. G. 1987. Carbohydrate, organic acid, and amino acid composition of bacteroids and cytosol from soybean nodules. Plant Physiol. 85:768-773.

Streit, W. R., Joseph, C. M., and Phillips, D. A. 1996. Biotin and other water-soluble vitamins are key growth factors for alfalfa root colonization by Rhizobium meliloti 1021. Mol. Plant-Microbe Interact. 9:330-338.

Wexler, M., Gordon, D., and Murphy, P. J. 1995. The distribution of inositol rhizopine genes in Rhizobium populations. Soil Biol. Biochem. 27:531-537.

Wood, M., and Stanway, A. P. 2001. myo-Inositol catabolism by Rhizobium in soil: HPLC and enzymatic studies. Soil Biol. Biochem. 33:375-399.

Yoshida, K., Aoyama, D., Ishio, I., Shibayama, T., and Fujita, Y. 1997. Organisation and transcription of the myo-inositol operon, iol, of $B a$ cillus subtilis. J. Bacteriol. 179:4591-4598. 